\title{
FEEDBACK BETWEEN ACCELERATOR PHYSICISTS AND MAGNET BUILDERS
}

\author{
S. PEGGS \\ Relativistic Heavy Ion Collider, Brookhaven National Laboratory, \\ Upton, New York 11973, USA
}

(Received 5 January 1996; in final form 5 January 1996)

The approach taken at Brookhaven National Laboratory in the RHIC project is described concerning the interplay between magnet design and construction and accelerator physics aspects. A set XXXXXXXXX.

Keywords: Magnet design; superconducting magnets.

\section{PHILOSOPHY}

Our task is not to record history but to change it. K. Marx (paraphrased)

How should Accelerator Physicists set magnet error specifications? In a crude social model, they place tolerance limits on undesirable nonlinearities and errors (higher order harmonics, component alignments, et cetera). The Magnet Division then goes away for a suitably lengthy period of time, and comes back with a working magnet prototype that is reproduced in industry.

A better solution is to set no specifications. Accelerator Physicists begin by evaluating expected values of harmonics, generated by the Magnet Division, before and during prototype construction. Damaging harmonics are traded off against innocuous harmonics as the prototype design evolves, lagging one generation behind the evolution of expected harmonics. Finally, the real harmonics are quickly evaluated during early industrial production, allowing a final round of performance trade-offs, using contingency scenarios prepared earlier. 
This solution assumes a close relationship and rapid feedback between the Accelerator Physicists and the magnet builders. What follows is one perspective of the way that rapid feedback was used to "change history" (improve linear and dynamic aperture) at RHIC, to great benefit. The busy reader may wish to skip directly to the GOLDEN RULES section that concludes this paper.

\subsection{Notation}

Satisfactory quench performance is assumed, and the body field of a dipole is written

$$
B_{y}+i B_{x}=B_{0}\left[1+10^{-4} \sum_{n}\left(b_{n}+i a_{n}\right)\left(\frac{x+i y}{R_{0}}\right)^{n}\right]
$$

where $R_{0}=\frac{5}{8} R_{\text {coil }}$ is the RHIC reference radius.

\section{RHIC OVERVIEW}

\subsection{Magnet Construction and Correction Schemes}

The copious and easy RHIC magnets are built in industry, while the specialized critical magnets are built "in house". Cryostats with multiple magnet components, such as the Corrector-Quad-Sextupole (CQS) packages, are assembled in house.

One arc half cell consists of two cryostats - an arc dipole (as delivered), and a CQS. All of the arc dipoles and quads built by Northrop-Grumman (NG), and the sextupoles and trim quadrupoles built by Everson, will have been delivered by the end of January 1996. Arc correctors are either 1 layer (dipole), or 4 layer (dipole, normal or skew quad, octupole, decapole). In house corrector production is almost finished. A single Interaction Region (IR) triplet cryostat will contain 3 quadrupoles, 1 dipole, and 3 four layer correctors, for both Blue and Yellow rings. All IR components are built in house. The correctors and quads are then assembled into CQ cold masses. Finally, each (very large) triplet cryostat is assembled, in the tunnel, from 6 CQ cold masses and 2 IR dipoles.

Closed orbit correction uses a combination of single and dual plane BPMs and dipole correctors. Global linear decoupling uses 2 skew quad families to minimize the closest approach of betatron tunes. Local linear decoupling uses 
2 skew quad families, and 12 individual skew quads, one in every triplet. The global chromaticity is corrected using two families of arc sextupoles, while global skew chromaticity correction uses normal and skew sextupole layers in the triplet correctors. Transition is jumped by reversing the excitation polarity of quad correctors, in a novel "linear transition jump" scheme. The injection tune spread can be tuned by the arc octupole and decapole corrector layers. This appears to be unnecessary (see below). Betatron tune spread for Landau damping can be driven by octupoles in zero dispersion regions. Triplet magnetic correction is complicated, using 8 tuning shims in each IR quad, "golden" quad selection, triplet sorting, and dead reckoned triplet corrector excitations. See elsewhere in these proceedings. ${ }^{1}$

\subsection{Tracking and Simulation}

Almost all RHIC simulations use a public release of TEAPOT, ${ }^{2}$ allowing us to file bug reports and request modifications. However, several RHIC idiosyncrasies (such as common dipoles, and skew chromaticity correction) must be included in an accurate model. They are introduced by running locally developed "filter" programs, which both read and write a fully instantiated TEAPOT machine file that represents RHIC. It is unfortunate that this file format is unique to TEAPOT, and that the "Standard (MAD) Input Format" is not rich enough for these purposes. Therefore, we fully support the proposal (in these proceedings) for a "Standard Machine Format" that many standard accelerator codes would read. ${ }^{3}$

Before the industrial production of each magnet style began, the dominant use of TEAPOT was to answer "what if .." design questions. Emphasis was placed on linear aperture measures, such as tune spread, smear, and correction performance, since the answers come much faster in this way. Only occasionally was the dynamic aperture tracked, to confirm design improvements suggested by linear aperture investigations. Studies showed that systematic harmonic errors usually dominate random errors. Potential differences between industrial and prototype systematics were incorporated in the expected harmonics by quoting THREE numbers per harmonic, instead of the usual TWO: $\left\langle b_{n}\right\rangle_{e}, \sigma\left(b_{n}\right)_{e}$ and $\Delta b_{n}$ are the systematic, random, and systematic range components. That is, a systematic harmonic measured over an ensemble of production lines is expected to lie in the range

$$
\left\langle b_{n}\right\rangle_{e}-\Delta b_{n}<\left\langle b_{n}\right\rangle<\left\langle b_{n}\right\rangle_{e}+\Delta b_{n}
$$


A typical question, for example, was "What if $\left\langle b_{4}\right\rangle$ in the arc dipoles at injection is at the extreme end of its expected range?"

During the very early industrial production of arc dipoles and quadrupoles, TEAPOT was used to evaluate real harmonics. As discussed below and elsewhere in these proceedings, ${ }^{4}$ a small number of contingency plans were invoked in the production line. Now that we are very late in the production phase, the actual harmonic distributions have (mostly) replaced the expected harmonics in our simulations. Actual distribution values of other parameters are also used, such as an rms CQS misalignment error of $\sigma=0.25$ [mm]. In the future, TEAPOT will move towards a controls modeling role, to predict the behavior of RHIC, warts and all.

\section{ARC DIPOLES}

The four harmonics of greatest concern to RHIC arc dipoles turned out to be

(1) random variations of the Integral Transfer Function (ITF) at storage

(2) systematic body skew quadrupole, $\left\langle a_{1}\right\rangle$, at storage

(3) systematic integrated sextupole, $\left\langle b_{2}\right\rangle$, at storage, and

(4) systematic body decapole, $\left\langle b_{4}\right\rangle$, at injection.

Ironically, these harmonics presented themselves as historical challenges from the highest harmonic order down, in reverse order of the list.

When the first few production dipoles were cold tested, they showed injection decapole harmonics consistent with $\left\langle b_{4}\right\rangle \approx\left\langle b_{4}\right\rangle_{e}+\Delta b_{4}$ using the values shown in Table I. If nothing was done the injection tune spread of a gold beam would be unacceptably large. Three potential production line responses had already been jointly prepared and evaluated, which modified the current distribution very slightly without modifying the iron cross section, by adjusting the thickness of either the mid-plane caps, or one or both of the coil wedges. ${ }^{4}$ We asked NG to change the thickness of the mid-plane caps from 0.006 to 0.004 of an inch. This traded off greatly improved $\left\langle b_{4}\right\rangle$ at injection with a slight increase in $\left\langle b_{2}\right\rangle$ (sextupole) at storage that is easily compensated using the chromaticity sextupoles. This modification took hold at arc dipole number 20, without interrupting magnet production. Later on, at magnet number 106, we returned to 0.006 inch mid-plane caps, while simultaneously increasing the size of one of the coil wedges by 0.0025 of an inch. The cost to the project was zero, since parts were already on hand, the construction procedure was unchanged, and the wedge dimension 
TABLE I Arc dipole body harmonics at injection (660 A) and storage $(5 \mathrm{kA})$, summarizing all cold measurement data available on October 4, 1995. Subscript $e$ implies "expected" values. Lack of space prevents inclusion of the important multipoles at the dipole lead and return ends

\begin{tabular}{crrrrrrrrrr}
\hline$n$ & \multicolumn{1}{c}{\langle\rangle$_{e}$} & $\Delta b$ & $\sigma(b)_{e}$ & \multicolumn{1}{c}{$\langle b\rangle$} & $\sigma(b)$ & $\langle a\rangle_{e}$ & $\Delta a$ & $\sigma(a)_{e}$ & $\langle a\rangle$ & $\sigma(a)$ \\
\hline $660 \mathrm{~A}$ & & & & & & & & & & \\
1 & 0.0 & 0.4 & 0.8 & 0.1 & 0.2 & 0.0 & 1.0 & 1.3 & 0.7 & 1.5 \\
2 & -2.5 & 4.0 & 2.3 & -2.2 & 2.0 & 0.0 & 0.4 & 0.5 & 0.0 & 0.2 \\
3 & 0.0 & 0.2 & 0.3 & -0.0 & 0.1 & 0.0 & 0.3 & 1.0 & 0.0 & 0.4 \\
4 & 0.0 & 1.0 & 0.6 & -0.4 & 0.6 & 0.0 & 0.1 & 0.2 & -0.0 & 0.1 \\
5 & 0.0 & 0.0 & 0.1 & 0.0 & 0.0 & 0.0 & 0.1 & 0.3 & 0.0 & 0.2 \\
6 & -0.1 & 0.2 & 0.2 & -0.2 & 0.1 & 0.0 & 0.0 & 0.1 & -0.0 & 0.0 \\
7 & 0.0 & 0.0 & 0.1 & -0.0 & 0.0 & 0.0 & 0.0 & 0.1 & -0.0 & 0.1 \\
8 & 0.3 & 0.1 & 0.1 & 0.2 & 0.1 & 0.0 & 0.0 & 0.1 & -0.0 & 0.0 \\
9 & 0.0 & 0.0 & 0.1 & 0.0 & 0.0 & 0.0 & 0.0 & 0.1 & 0.0 & 0.0 \\
10 & -0.5 & 0.0 & 0.1 & -0.6 & 0.0 & 0.0 & 0.0 & 0.1 & -0.0 & 0.0 \\
& & & & & & & & & & \\
$\mathrm{kA}$ & & & & & & & & & & \\
1 & 0.0 & 0.4 & 0.8 & 0.1 & 0.2 & -2.5 & 0.5 & 1.3 & -1.3 & 1.5 \\
2 & -2.8 & 4.0 & 2.3 & -1.7 & 1.6 & 0.0 & 0.4 & 0.5 & -0.1 & 0.2 \\
3 & 0.0 & 0.2 & 0.3 & 0.0 & 0.1 & 0.0 & 0.3 & 1.0 & -0.4 & 0.4 \\
4 & 0.5 & 1.0 & 0.6 & 0.1 & 0.6 & 0.0 & 0.1 & 0.2 & -0.0 & 0.1 \\
5 & 0.0 & 0.0 & 0.1 & -0.0 & 0.0 & 0.0 & 0.1 & 0.3 & -0.0 & 0.2 \\
6 & 1.2 & 0.2 & 0.2 & 1.1 & 0.1 & 0.0 & 0.0 & 0.1 & -0.0 & 0.0 \\
7 & 0.0 & 0.0 & 0.1 & -0.0 & 0.0 & 0.0 & 0.0 & 0.1 & 0.0 & 0.1 \\
8 & 0.3 & 0.1 & 0.1 & 0.2 & 0.1 & 0.0 & 0.0 & 0.1 & -0.0 & 0.0 \\
9 & 0.0 & 0.0 & 0.1 & 0.0 & 0.0 & 0.0 & 0.0 & 0.1 & 0.0 & 0.0 \\
10 & -0.5 & 0.0 & 0.1 & -0.6 & 0.0 & 0.0 & 0.0 & 0.1 & -0.0 & 0.0 \\
\hline & & & & & & & & & & \\
\hline
\end{tabular}

modification was already foreseen in the legal contract. As a consequence, the decapole correctors do not need power supplies, a considerable cost saving.

Systematic body skew quadrupole at storage, $\left\langle a_{1}\right\rangle$, comes from the vertical offset of the dipole cold mass in its cryostat, causing the field that leaks from the yoke iron to the cryostat to have an asymmetric distribution. Although the early value of $\left\langle a_{1}\right\rangle \approx-2.5$ was near the center of its expected range, a safe level, we later on requested that NG simply put the heavier yoke half on the bottom. This cost-neutral modification, which resulted in $\left\langle a_{1}\right\rangle \approx-1.3$, was concurrent with a total yoke weight increase requested for other reasons. ${ }^{4}$

There were several other minor arc dipole interventions. ${ }^{1,4,5}$ For example, close cooperation between RHIC and NG lead to substantial reductions in the rms variation of the field angle along the dipole. Similarly, attention focused 


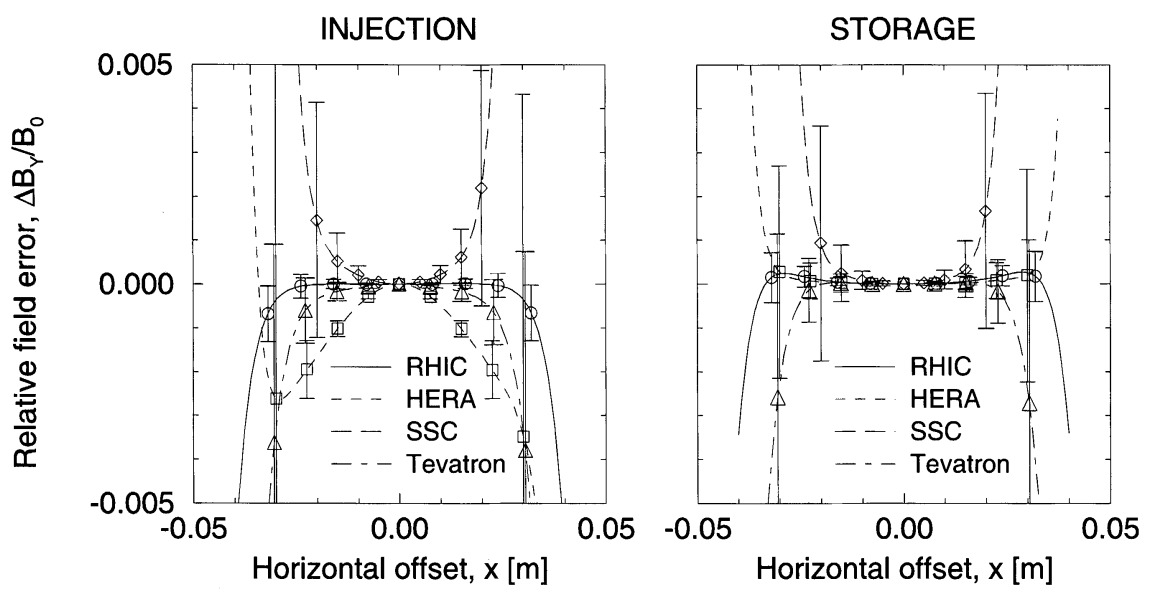

FIGURE 1 Dipole field profiles, as measured for RHIC, HERA, Tevatron, and as expected for the SSC. Curves and error bars represent systematic and random harmonics. At injection, HERA is hampered by persistent currents, while the Tevatron is dominated by an intentional $b_{8}$. RHIC systematics benefit from a high injection field, and randoms benefit from small filaments. At storage, RHIC shows modest $b_{2}$ due to iron proximity, still with very good randoms.

on coil size variations at the 2 or $3 \mu \mathrm{m}$ level, when trends in $\left\langle b_{4}\right\rangle$ and $\left\langle a_{4}\right\rangle$ $(\approx 0.2$ unit) were noticed, and traced back to the need to clean NG tooling.

\subsection{Arc Dipole Field Profiles}

Figure 1 demonstrates the high quality of RHIC dipoles, by comparing their field profiles with those from other super-conducting machines, out to the appropriate coil radius. This is a fair comparison for RHIC, HERA, and the Tevatron, which have coil diameters of 80.0, 75.0, and $76.2 \mathrm{~mm}$. The SSC suffers in this comparison, not only because it has a coil diameter of $50 \mathrm{~mm}$, but mainly because the feedback loop between Accelerator Physics and Magnet Division was never (satisfactorily) closed. Note the large SSC random harmonics. It would be interesting to add current LHC expectations to these plots.

\subsection{Arc Dipole Integral Transfer Function \& Sorting}

At RHIC, the possibility of sorting on arc dipole locations was originally held in reserve as a "back pocket" contingency against unexpected problems. HERA sorted their dipoles, for example, on $b_{2}$, on the ITF, and on intermagnet pipe welding misalignments. It was only after practice showed that 
TABLE II ITF sorting statistics for arc dipoles at storage, in BLUE and YELLOW rings

\begin{tabular}{lrrr}
\hline & & BLUE & YELLOW \\
\hline ITF average & {$[\mathrm{Tm} / \mathrm{kA}]$} & 6.41825 & 6.41822 \\
ITF standard deviation, $\sigma(I T F)$ & {$[\mathrm{Tm} / \mathrm{kA}]$} & 0.00203 & 0.00181 \\
(Standard deviation)/average & & 0.00032 & 0.00028 \\
Corrector current for $1 \sigma(I T F)$ error & {$[\mathrm{A}]$} & 1.75 & 1.56 \\
\hline
\end{tabular}

pipe misalignments were small enough (not trivially obvious!) that we felt free to sort on storage ITF. An arc dipole ITF error causes the neighboring horizontal closed orbit corrector to have an excitation offset. Two dipoles usually contribute to one corrector. Hence, high and low ITF dipoles are matched in pairs across horizontal dipole correctors, in order to reduce the offset by a few Amps. Since only 1 in 10 dipoles is measured cold, the measurement accuracy (including warm to cold correlation) of $\approx 0.4 \sigma$ is an important parameter. Only dipoles far out in the distribution tails are sorted. Table II records relevant statistics for all arc dipoles available in October 1995. Since most dipole correctors have 25 Amp power supplies, and any one may be upgraded to $50 \mathrm{Amps}$, the advantages of ITF sorting are largely academic.

\section{CORRECTOR-QUADRUPOLE-SEXTUPOLE (CQS) PACKAGES}

Similar (but fewer) anecdotes can be told about field harmonics in RHIC arc quads. Here again the focus was on allowed and semi-allowed systematic harmonics, such as body octupole $\left\langle b_{3}\right\rangle$, which was trimmed very early in production. However, the REAL challenge has been to assemble CQS magnets so that their components can be confidently placed close to the design orbit. The rigid composite cold mass must be free to shrink and expand longitudinally $\left(\Delta L / L \approx 4 \times 10^{-3}\right)$ during thermal cycling. At the same time, the transverse motion must be severely constrained. For example, the quad field center must be placed with sub-millimeter accuracy, especially vertically, so that polarized protons can be successfully accelerated. Alignment and location are closely related problems which must be distinguished. First, the three magnets and the BPM in each CQS must be aligned during assembly into a rigid and very straight line. Second, the 
magnetic field centers (after assembly) must be located, relative to fiducials outside the cryostat that are available to surveyors.

Several systematic and random problems at the $0.1 \mathrm{~mm}$ level have been addressed. "Springs" made of G-10 plastic, installed in the support posts, push the cold mass transversely while allowing free longitudinal motion. The welding sequence is carefully choreographed to balance "curling" distortions against each other. A colloidal cell optical technique is used to locate the quad field center of a fully assembled warm package, relative to external fiducials. ${ }^{5}$ All these activities required significant learning curves. Individual CQS magnets coming off the assembly line are evaluated for $2 \sigma$ deviations from distribution norms, and for violations of absolute limits, on various quantities, such as the quad field angle. These data are used by the surveyors to install the CQS so that quad and sextupole field centers are on the design trajectory, and the integrated quad field angle is zero. Table III records the alignment statistics at the time of writing, with about half of the CQS magnets fully assembled. BPM and corrector offsets from the design trajectory indicate the CQS curl. The apparent offset of the colloidally measured quad field center, relative to the mechanically measured center, gives an internal consistency check on quad misalignment.

\section{GOLDEN RULES}

Caveat emptor: while the following golden rules of RHIC magnet design and modeling are mostly general in nature, there is no guarantee that a particular rule applies to a specific machine - such as the LHC.

TABLE III CQS alignment statistics using colloidal cell measurements of the quad field center, justifying the simulation assumption that the RMS quadrupole misalignment error $\sigma=0.25$ [mm]

\begin{tabular}{|c|c|c|c|}
\hline Quantity & Units & Mean & S.D. \\
\hline Quadrupole field angle & [mrad] & -1.72 & 0.30 \\
\hline Sextupole field angle & [mrad] & -0.52 & 0.35 \\
\hline \multirow{2}{*}{$\begin{array}{r}\text { S.D. of } 5 \text { colloidal measurements per quad, horizontal } \\
\text { vertical }\end{array}$} & [in] & -0.014 & 0.003 \\
\hline & [in] & 0.015 & 0.002 \\
\hline (Colloidal - mechanical) amplitude & [mm] & 0.20 & 0.07 \\
\hline Corrector offset amplitude & [mm] & 0.51 & 0.15 \\
\hline BPM offset amplitude & {$[\mathrm{mm}]$} & 0.57 & 0.28 \\
\hline
\end{tabular}




\subsection{Magnet Design}

With careful control of superconducting cable tolerances, random harmonics are relatively unimportant - systematics dominate. Contemporary 2-D field codes used for magnet cross section design miscalculate the dominant systematic harmonics of a perfectly constructed magnet by less than "a few units". Saturation effects, $\left\langle b_{n}\right\rangle$ vs $I$, may be controlled to better than a few units during the design phase by resizing and moving holes in the iron yoke. Prototype cross section iterations are needed for better design accuracy than this. Systematic harmonics in "build to print" industrial magnets differ from those in prototype magnets by a smaller (but still significant) amount, simply due to realistic tooling tolerances. "Delta function" multipoles from the coil return and coil lead geometries at the magnet ends also need attention (minimization) during the design phase.

\subsection{Magnet Modeling - Tracking and Simulation}

Arc dipole errors dominate at injection, when the unnormalized emittance is large and the collision optics are detuned. Interaction region triplet quadrupole errors dominate at storage, when small $\beta^{*}$ values make the beam very large there. In general, model each magnet by smoothly distributed body harmonics, plus independent $\delta$-function multipoles at each end. It is not necessary to attach $\Delta b_{n}$ parameters to magnet end multipoles. Short magnets ("constant" $\beta$ and small $\Delta \phi$ ) may be modeled and designed as integrated lumps.

\subsection{Before Industrial Production}

Parameterize each body harmonic with 3 quantities: $\left\langle b_{n}\right\rangle, \sigma\left(b_{n}\right)$, and the "plausible systematic range" $\Delta b_{n}$, which represents realistic variations of systematic harmonics over an ensemble of production lines. Evaluate the potential threat of each $\Delta b_{n}$, using the best "expected" values provided by the magnet builders. Prepare production line response scenarios (eg, shim or cap dimension modifications) for the most threatening $\Delta b_{n}$ terms. Evaluate possible side effects on other harmonics.

\subsection{During Industrial Production}

As soon as statistically significant data for $\left\langle b_{n}\right\rangle$ (and $\left\langle a_{n}\right\rangle$ ) are available, apply any appropriate responses. Monitor systematic harmonics (and other data) for 
trends during the entire production run. Use copious early data to understand warm-cold harmonic measurement correlations, in order to reduce expensive cold testing.

\subsection{Arc Dipole}

Watch allowed harmonics $\left\langle b_{2}\right\rangle$ and $\left\langle b_{4}\right\rangle$ at injection. Watch the Integral Transfer Function (ITF) [Tm/kA], $\left\langle a_{1}\right\rangle$, and $\left\langle b_{2}\right\rangle$ at storage. Watch for (mechanical) field angle variations along the length of the dipole.

\subsection{Arc CQS or Spool Piece}

Watch the allowed quadrupole harmonics. Avoid curling distortions during assembly by learning the least damaging welding choreography. Measure the quadrupole field center relative to external surveying fiducials, after all welding. Evaluate the mechanical constraints of quadrupoles against random transverse movements (during magnet transportation, cool down, thermal cycling, and quenching). Measure the systematic components of transverse quadrupole movements, and compensate during installation/surveying.

\section{Acknowledgements}

I would like to thank my friends and colleagues on the "Magnet Acceptance Committee", for the direct and indirect contributions that they made to this paper.

\section{References}

[1] J. Wei, "Error Compensation in Insertion Region Magnets", these proceedings.

[2] L. Schachinger and R. Talman, "TEAPOT, Thin Element Tracking Program for Optics and Tracking”, Particle Accelerators, 1987.

[3] N. Malitsky, R. Talman, et al., "A Proposed Flat Yet Hierarchical Accelerator Lattice Object Model", these proceedings.

[4] R. Gupta, "Estimating and Adjusting Field Quality in Superconducting Magnets", these proceedings.

[5] RHIC Magnet Division, "Papers Submitted to 1995 PAC in Dallas, and to the International Conference on Magnet Technology, Tampere, 1995", available as a compendium from RHIC, BNL, Upton, NY. 